\title{
Espacio de aprendizajes y apoyo escolar. Un recorrido sobre el trabajo extensionista en la Biblioteca Popular Pocho Lepratti
}

\author{
Paula Castagna \\ programascomunitarios@unr.edu.ar
}

Lucila Nepote

programascomunitarios@unr.edu.ar
Secretaría de Extensión Universitaria de la Universidad Nacional de Rosario, Argentina.
A 100 años de la Reforma Universitaria de 1918 /

Desafíos de gestión

RECEPCIÓN: 29/06/17

ACEPTACIÓN FINAL: 23/09/17

\section{Resumen}

El Área de Programas Comunitarios de la Secretaría de Extensión Universitaria de la Universidad Nacional de Rosario (UNR) coordina el "Espacio de Aprendizajes" en la Biblioteca Popular Pocho Lepratti junto a voluntarios de diversas unidades académicas y residentes de cátedras de prácticas no formales.

En este texto daremos cuenta del modo en que se construyó este espacio y del recorrido realizado, poniendo en visibilidad los replanteos teórico-prácticos que se fueron dando desde el comienzo, en 2012, hasta 2016.

En este espacio, destinado a los niños y niñas de las escuelas primarias de la zona, tomamos como ejes de trabajo lo histórico-social, lo artístico-cultural, lo pedagógico y la salud en general, desde una perspectiva de promoción de los derechos.

Nos propusimos una doble misión: por un lado, focalizar acciones sociales comprometidas con la comunidad de la que somos parte y, por el otro, establecer espacios de aprendizajes donde los contenidos son definidos por el trabajo que se hace en el territorio, con acompañamiento del proceso escolar y focalizando en las tareas y actividades de acuerdo con sus necesidades e intereses.

\section{Resumo}

A Área de Programas Comunitários de Secretaría de Extensão Universitária da UNR coordena o "Espaço de Aprendizagens" na Bibliotéca Popular "Pocho Lepratti”, junto com voluntários de diversas unidades acadêmicas e residentes de cátedras de práticas não formais.

Neste texto, tentaremos dar uma conta do curso do espaço e da sua construção com base nas circunstâncias e nos repensamentos teórico -práticos que ocorreram desde o início até 2012 até 2016.

Está destinado às crianças das escolas primárias da região. Tomamos como eixos o histórico-social, o artístico-cultural, o pedagógico e a saúde em geral, desde uma perspetiva de promoção dos directos.

Proponemos o espaço com uma dupla missão: por um lado, uma atividade que otorgue a possibilidade de focalizar ações sociais comprometidas com o entorno e com a comunidade da qual fazemos parte; por outro lado, proponer um espaço de construção de aprendizagens onde os conteúdos são estabelecidos pelo trabalho que se faz em território, acompanhando o processo escolar, focalizando nas tarefas e atividades de acordo com as suas necessidades e interesses.

\section{Palabras clave}

- Infancias

- Aprendizajes

- Educación popular

- Extensión crítica

- Cambio social
Palavras-chave

- Infância

- Aprendizagens

- Educação popular

- Extensão crítica

- Câmbio social de este artículo

Castagna, P. y Nepote, L. (2017). Espacio de aprendizajes y apoyo escolar. Un recorrido sobre el trabajo extensionista en la Biblioteca Popular Pocho Lepratti. Revista +E versión en línea, 7(7), 214-224.

Santa Fe, Argentina: Ediciones UNL. 


\section{Introducción}

El Área de Programas Comunitarios de la Secretaría de Extensión Universitaria de la UNR comprende la coordinación de las actividades desarrolladas en el ámbito de instituciones comunitarias e incluye actividades curriculares y extracurriculares, a saber:

- Impulsar la participación de estudiantes y docentes en actividades de interés comunitario.

- Establecer convenios de cooperación, con organismos oficiales y otros, para la realización de actividades conjuntas.

- Coordinar con Unidades Académicas la realización de actividades interdisciplinarias en la comunidad.

- Gestionar, ante otros organismos, financiamiento para proyectos de actividades comunitarias.

- Coordinar criterios de regulación y seguimiento de actividades en la comunidad.

- Promover la presentación de proyectos de actividades comunitarias.

Ante estos lineamientos y objetivos, en el marco de un convenio que se realizó entre la Universidad Nacional de Rosario y la Comisión Nacional de Bibliotecas Populares en el año 2012, desde el Área de Programas Comunitarios nos acercamos a la Biblioteca Popular Pocho Lepratti con el objetivo de conocer la historia y la realidad institucional para así poder coordinar acciones en conjunto. A partir de este hecho, el área comenzó a coordinar el "Espacio de aprendizajes y apoyo escolar" en la biblioteca, junto a estudiantes voluntarios de diversas unidades académicas y residentes de algunas cátedras de prácticas no formales.

En este texto intentaremos dar cuenta del recorrido del espacio y la construcción del mismo a partir de las circunstancias y replanteos teórico-prácticos que se fueron dando desde el comienzo hasta el año 2016

Para hacer una referencia histórica:

"es necesario reconocer que «extensión universitaria» es un referente nodal de la genealogía del movimiento de Reforma Universitaria ocurrido en América Latina en la primera mitad del siglo XX, que tuvo en los sucesos de Córdoba de 1918 uno de sus hitos (Carlevaro, 2002; Tünnermann, 2000). Y desde esa raíz, en muchas universidades del continente, 'extensión' ha sido un significante que ha permitido articular proyectos político-académicos capaces de disputar los modos hegemónicos de hacer universidad, procurando vincular los procesos de enseñanza y creación de conocimiento con los grandes problemas nacionales y las necesidades de los sectores populares de la sociedad". (Tommasino y Cano, 2016:8)

Con esta afirmación, sostenemos que hay que salir de la Universidad e inmiscuirse en los territorios, en los barrios y en los espacios populares donde se construye una posibilidad de desarrollo con los otros, con los iguales y con los distintos, con todo lo que se tiene y con lo que falta. Para dejar de ser claustros cerrados en sí mismos y poder integrar la práctica de extensión con las de docencia e investigación, para poder hacer partícipes a los estudiantes en los cambios que la comunidad procura y demanda, siendo parte, ya que la Universidad tiene que estar atenta a las acciones sociales y en particular anticiparse a las necesidades para que las demandas específicas tengan respuesta por parte de un actor social, que es la universidad comprometida con el medio y el contexto.

\section{¿Qué es la Biblioteca Popular Pocho Lepratti?}

La Biblioteca Popular Pocho Lepratti se emplaza en el Barrio La Tablada en el sur de la ciudad de Rosario, provincia de Santa Fe. En la actualidad el barrio presenta una serie de situaciones problemáticas vinculadas, de modo general, a un aumento de la conflictividad y la violencia que impactan fuertemente en jóvenes que se encuentran en situación de vulnerabilidad social. Situaciones vinculadas a las disputas por territorios marcados por el narcotráfico, la falta de oportunidades, desigualdades sociales, niveles de corrupción y un alto descreimiento. No obstante, hay que destacar que gran parte de los vecinos del barrio siguen creyendo y trabajando por la articulación e integración de los espacios, el compromiso por la educación y la salud, participando y trabajando por el barrio y la comunidad en general. Pero esta situación se remonta al surgimiento del barrio, tal como lo plantean Koldorf, Castro y Tessore en su trabajo "El barrio como espacio de interacción social":

"Hasta la mitad de los años "70 las familias de ambos sectores sociales, aunque sostenían concepciones del mundo disímiles, establecieron una red de vínculos sociales e institucionales, que actuaban como un verdadero tejido contenedor. Con la implementación del sistema económico neoliberal a nivel nacional y, teniendo en cuenta el proceso de reconfiguración que adquiere el Estado, especialmente durante los '90, y las transformaciones barriales que se produjeron en este período en Tablada, estas relaciones se fueron desarticulando; disminuyeron lugares y entramados de sociabilidad e intercambio; al tiempo que van surgiendo confrontaciones y fricciones entre los distintos sectores étnicos y de clase". (2008:2)

"El barrio Tablada se vio particularmente afectado con el cierre y quiebra de numerosas fuentes de trabajo; frigoríficos de la zona que se reconvierten con achicamiento de personal; el desmantelamiento de los ferrocarriles, que incluyó a varias generaciones de asalariados; el cese de funciones del puerto y del Ministerio de Obras Públicas (MOP). 
La incertidumbre, desconfianza y escepticismo en el porvenir, la falta de pertenencia y de vínculos entre vecinos y allegados transformó el espacio barrial y sus sentidos y representaciones. Los vínculos que solían unir a los habitantes de los asentamientos irregulares con los vecinos del barrio y con el resto de la sociedad, en los diversos períodos de una Argentina en crecimiento socioeconómico, a través de su participación intermitente en el mercado de trabajo y en el sistema escolar, hoy se vislumbran severamente dañados y fragmentados". (7)

Estas situaciones se enmarcan en un presente en el cual la lógica del mercado se erige paulatinamente como principio central en la regulación de los lazos sociales, subalternizando al Estado y la comunidad. En este marco, son notorias las dificultades de las instituciones, entre ellas la escuela, las organizaciones sociales y la Universidad, para poder dar respuesta a determinadas transformaciones sociales.

En este horizonte de crisis, el carácter homogeneizador de la escuela se presentaría estéril para poder incluir o trabajar con determinadas diferencias ya sean sociales, de género o culturales. Sin embargo, se considera que estas instituciones seguirían ocupando un lugar central en lo referente a la construcción de ciudadanía y subjetividades. En este sentido, Duschatzky sostiene:

"En tiempos estables y de clara hegemonía de los Estados Nación, la relación entre jóvenes, escuela y pobreza se planteaba en términos de posibilidad: mediante la escuela, los pobres pueden acceder a nuevas posiciones sociales; o de crítica: la escuela reproduce las desigualdades sociales existentes". (2003:1)

Dentro de este contexto y luego de los sucesos ocurridos en todo el país en el año 2001, surge la Biblioteca Popular "Pocho Lepratti". Es una asociación civil sin fines de lucro inaugurada el 18 de octubre de 2002. Lleva el nombre de Claudio "Pocho" Lepratti, un militante social, exseminarista cristiano, que realizó un intenso trabajo comunitario dirigido a los niños y jóvenes en situación de vulnerabilidad social en la ciudad de Rosario. Fue asesinado en las jornadas de diciembre de 2001 cuando increpaba a la policía desde el techo de la escuela donde trabajaba en el comedor para que no dispararan porque allí solo había chicos comiendo. A raíz de esta acción, el cantante argentino León Gieco le dedicó su tema "El ángel de la bicicleta". La Biblioteca que hoy lleva su nombre se propone recordar su trayectoria y realizar acciones socioculturales desde la óptica de la educación popular, reivindicando la inclusión social desde la perspectiva de un proyecto y de un equipo de trabajo que propicia la participación, el protagonismo popular y la construcción de una ciudadanía plena.

Así es que la Biblioteca ha afirmado en estos 14 años su compromiso con el trabajo y el desarrollo comunitario, se ha propuesto como un espacio de cultura y educación popular, que apunta a recuperar experiencias y promover redes sociales; y como un espacio de comunicación comunitaria, que ha intentado siempre articular las problemáticas de interés de sus vecinos con el fin de contribuir a hacer del barrio un lugar solidario y organizado, donde sus habitantes puedan apropiarse de cada actividad y sentirse partícipes de cada logro. Como herramienta cultural al servicio del barrio propone talleres de arte, juegos, apoyo escolar, taller de expresión audiovisual, y actividades de programación cultural diversas (música, teatro, muestras, presentaciones). A esto se suma el funcionamiento de un Jardín de Infantes con salas de 2, 3 y 4 años, y del Centro de día destinado a los jóvenes del barrio.

\section{Primeros pasos...}

Uno de los talleres que la Biblioteca impulsó desde sus inicios fue el de apoyo escolar, pero en el momento en que la Secretaría de Extensión Universitaria (SEU) se acerca a la organización no se estaba desarrollando por no contar con los recursos necesarios. Ante la demanda de las escuelas y los vecinos, ya que muchos están marcados por condiciones de marginalidad que dificulta el acceso y el tránsito por la escolaridad, se propone en el año 2012 retomar con dicho espacio coordinado desde Área Comunitaria de la SEU.

Este fue el primer acercamiento con la institución y a partir de la firma del convenio marco de trabajo en conjunto se empezaron a diseñar las estrategias necesarias para retomar este taller. Primero se propuso un recorrido por las Escuelas primarias de la zona en el que, habiendo hablado previamente con la directora de cada institución, se acordó informar a los responsables de los niños y niñas con una nota que cada uno podía llevar a su casa. También se habló con las maestras ya que ellas son las que en el seguimiento diario pueden proponer que se recurra al apoyo escolar como soporte para algunos casos en particular. Al mismo tiempo fuimos haciendo una convocatoria desde la SEU en las distintas unidades académicas y en las redes sociales y contactos con los que contábamos para que tanto los estudiantes como los graduados pueda sumarse como voluntarios en este espacio, ya que consideramos necesaria la integración de los espacios populares y de las prácticas universitarias, pensando que el acceso a una práctica en terreno pone en relación lo académico con lo comunitario.

Así se fue planificando el taller que tiene como destinatarios a los niños y niñas de las escuelas primarias del barrio y se propuso llevarlo a cabo en tres encuentros semanales en un horario en el que puedan asistir tanto los niños y niñas del turno de la mañana como los de la tarde. Esta tarea se fue desarrollando junto con estudiantes y graduados de diversas unidades académicas, así como vecinos interesados en colaborar. En esta instancia se hizo notoria la demanda de este taller ya que casi inmediatamente empezaron a acercarse las madres, los padres o familiares a cargo a anotar a los chicos. El espacio en ese momento se focalizó 


\author{
El desafío fue proponer un espacio que \\ combine el juego, el arte y la comunicación, \\ y que permita representar y compartir \\ deseos e intereses de los participantes
}

en las tareas específicas que ellos traían de la escuela y en las actividades que iban demostrando una mayor dificultad, así como en el estudio para evaluaciones y confección de trabajos prácticos. Al mismo tiempo se realizaba un seguimiento del recorrido de cada materia o asignatura buscando propiciar el interés y la búsqueda de información en los textos, ya sea en los manuales de clase como en los libros de los que dispone la biblioteca, poniendo especial interés en el desarrollo comprensivo de los mismos, ya que consideramos que ese proceso les brindará herramientas para su posterior recorrido educativo, cultural y social.

El objetivo en ese momento se centró en brindar un acompañamiento a los niños y niñas en su proceso de aprendizaje desde una perspectiva no formal con la finalidad de que pudieran transitar el año escolar siendo partícipes de ese camino, así como fortalecer los vínculos entre la escuela, la familia y la biblioteca.

De allí que pensamos al espacio de apoyo escolar como un escalón más que puede acercar, en este proceso interactivo, a que niñas y niños sean formados en un espíritu crítico, capaces de tomar sus propias decisiones en las diversas situaciones que se presentan en el día a día, interviniendo en el lugar social del que forman parte.

Con la experiencia realizada en este taller durante la última mitad del año 2012 y analizando algunas situaciones particulares durante el desarrollo del mismo, consideramos enriquecedor generar, a partir de 2013, una instancia intermedia que brinde otras posibilidades, un espacio donde se puedan poner en práctica actividades desde una perspectiva lúdica, en la que se combine la participación activa, la integración y la comunicación. Lo que remarcamos es la importancia de acceder al conocimiento como eje que trasciende la pura acumulación de datos, tomando al conocimiento como herramienta para el tratamiento, el análisis y la transformación, tanto de su realidad como de sí mismos, como actores habilitados y habilitantes del cambio social y la construcción de una sociedad justa, equitativa y con posibilidades de acceso y participación. Para ello era necesario revertir actitudes individualistas y competitivas que se destacaban en el grupo por actitudes de cooperación y respeto.

El desafío fue proponer un espacio que combine el juego, el arte y la comunicación, y que permita representar y compartir deseos e intereses de los participantes, mostrar sus debilidades y fortalezas, comprometerse con un grupo, explorar y conocer aptitudes, involucrarse física e intelectualmente para fortalecer lazos, desde el respeto y la integración. Con ese propósito, tomamos como referencia a Winnicott y sus consideraciones sobre el juego como un logro en el desarrollo emocional, ya que sostiene que lo característico del juego es el placer y que esa satisfacción proviene del uso de símbolos. Propone al "juego como elaboración imaginativa en torno de las funciones corporales, a la relación con los objetos y con la angustia" ya que a través del juego como actividad creadora, el niño se ocupa de la realidad exterior y "puede expresar, mediante materiales externos, las relaciones y angustias internas" (2015:80).

Desde este lugar, podemos suponer en el juego la expresión de las identificaciones, de los afectos y el desarrollo de la capacidad de simbolización de las experiencias intersubjetivas. Así fue que en la planificación del taller para el año 2013 se propuso un espacio más al que llamamos "Espacio comodín" en el que tomamos como ejes de desarrollo lo histórico-social, lo artístico-cultural, lo pedagógico y la salud en general, propiciando un espacio creativo, un lugar de expresión donde descubrir lo propio, hablar de las culturas, de la música, promover actividades y gustos propios, lo que puede ser una ventana, un lugar de acceso a lo grupal donde disminuir las actitudes individualistas o violentas sosteniendo normas de convivencia.

A esta propuesta se sumaron practicantes de la carrera de Licenciatura en Psicopedagogía que estaban realizando las prácticas preprofesionales de la cátedra "Prácticas comunitarias psicopedagógicas". Este espacio consistió en abordar diferentes propuestas de trabajo entre las cuales podemos destacar: el juego del "dominó matemático" en donde las fichas se unen cuando el resultado de las cuentas coincide; los juegos del "Chancho va", "De la memoria", en el que las fichas coinciden cuando corresponden 


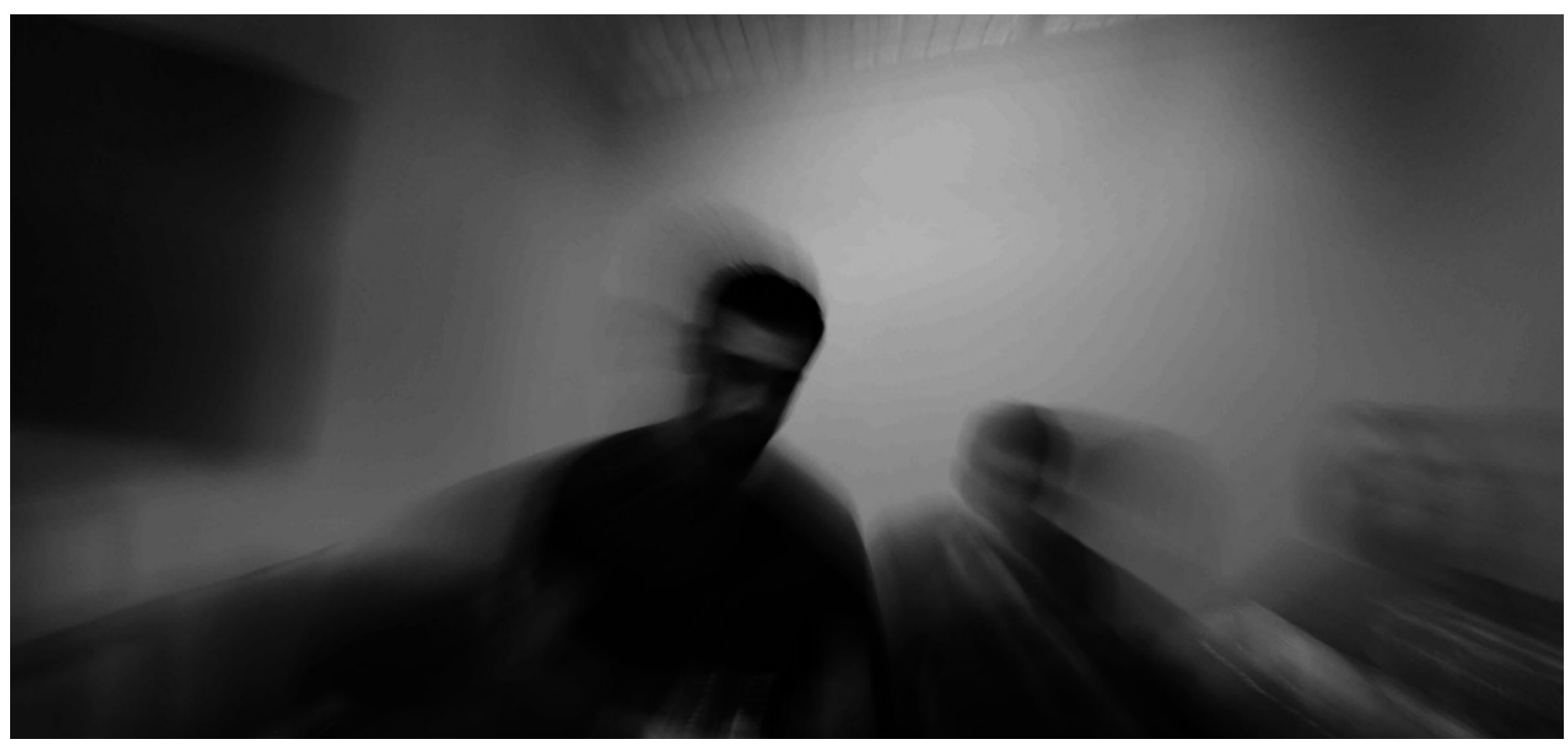

(C) Hugo Pascucci

los dibujos o la palabra con el dibujo, del "Bingo con palabras", todos realizados artesanalmente. También se generaron visitas de algunos de los profesionales o residentes del Centro de Salud Municipal que queda a pocas cuadras de la biblioteca. En estos encuentros se abordaron charlas en las que mediante el juego y la participación se intentó acercar algunas prácticas de prevención y asistencia de la salud. Además, contamos con la participación de un personaje secreto y misterioso que se hace llamar "el dibujante de la noche" y con el cual los chicos mantienen una correspondencia de cartas y dibujos; visitas de malabaristas o bailarines; visitas y charla con referentes del barrio o de la institución. Es decir, un espacio en el que se intenta combinar lo escolar y el aprendizaje formal con los saberes propios potenciando las aptitudes y haciendo hincapié en el cuidado y el respeto, tanto de uno mismo como del compañero, remarcando la idea de que el dominio de la palabra, el saber escribir, el saber leer, solamente tienen sentido si se traduce en una mejor lectura del mundo, una mejor lectura del contexto del hombre que le hace estar en el mundo, para poder transformarlo.

\section{Cuándo se hace extensión, ¿todo es extensión?}

El momento intermedio entre 2014 y 2015, el espacio-taller compartido con la organización, se complementa con los procesos de formación en extensión en el ámbito de la SEU. Participamos de congresos, jornadas y espacios activos de formación que nos acercaron a distintas problemáticas y planteos. Vislumbramos que las prácticas integrales y la capacidad de construcción dialógica debían ser parte de nuestra formación y nuestra práctica en y con la comunidad con la que día a día intentamos cambiar algo de la realidad para que sea menos agobiante.

El reflejo de estos planteos los podemos ejemplificar en la implementación de actividades en conjunto entre la universidad y la Biblioteca, al ampliar el espacio de acción conjunta, de visibilización de estas interacciones, de prácticas que trascienden las aulas o los espacios físicos de la universidad. Destacamos los programas de radio, cierres de la semana de la extensión, evaluaciones de proyectos y programas universitarios, participación en congresos y charlas, producción de libros, jornadas en defensa de la educación pública, entre otros.

Fue de la mano de estos nuevos espacios de formación y trabajo en conjunto con la Biblioteca, que se comenzó a implementar este "otro momento", el espacio comodín, como un lugar de expresión, de trabajo distendido, de momento de aprendizaje diferente. Fue una fase intermedia donde pudimos plantear un modo distinto de construcción de saberes, de intercambio de historias, de conocimiento mutuo.

Fue sostenido y pensado como "otro momento" porque veíamos necesario ampliar las estrategias sin abandonar el acompañamiento formal de la escuela.

Al interior del taller podemos destacar que se condensó un espacio común de participación lúdica y promoción de derechos, 
que compartimos historias de vidas atravesadas por desigualdades, desafíos, por crecimientos e incógnitas, por violencia y dificultades, por saberes en juego, por decisiones difíciles... ¿Cuál es el planteo o el límite? ¿Son estas actividades de extensión? ¿Cuándo se hace extensión, todo es extensión? En este sentido debemos destacar dos tendencias que pueden identificarse con referencia a la conceptualización de la práctica extensionista; una que considera que todas las actividades que la universidad hace en el medio son "extensión", donde predomina aquí el modelo transferencista; la otra tendencia que desarrollan Tommasino y Cano es la que articula la tradición pedagógica del movimiento de la educación popular latinoamericana (freiriana) y de la investigación-acción-participación (falsbordiana). "Aquí existe una preocupación explícita por la conceptualización de la extensión y su diferenciación de otro tipo de actividades universitarias en el medio" (2016:12).

Este es el modelo de extensión crítica. Vemos que ambas corrientes se mantienen hasta el presente, con algunas variantes, sobre todo a nivel metodológico. Todos estos procesos y tendencias conviven y se combinan haciendo de la vinculación con el medio y la extensión un campo sumamente heterogéneo, en el que debemos posicionarnos y fundamentar nuestras prácticas y experiencias. Al sostener prácticas transformadoras, asumimos los objetivos que la extensión crítica plantea y considera dialécticamente relacionados y en concordancia con la democratización del conocimiento en un proceso educativo no estereotipado. De estos objetivos:

"El primero se vincula con la formación de los universitarios y la posibilidad de establecer procesos integrales que rompan con la formación profesionalista alejada de un criterio de compromiso social de los graduados universitarios. La extensión concebida como un proceso crítico y dialógico se propone, en cambio, trascender la formación exclusivamente técnica que genera la universidad «fabrica de profesionales» (Carlevaro, 2010) y alcanzar procesos formativos integrales que generen universitarios solidarios y comprometidos con los procesos de transformación de las sociedades latinoamericanas. En segundo lugar, en su dimensión política, esta perspectiva de la extensión se propone contribuir a los procesos de organización y autonomía de los sectores populares subalternos intentando aportar a la generación de procesos de poder popular". (Tommasino y Cano, 2016:15)

Ante estos recorridos, vivencias y perspectiva que nos invitaban a replantearnos la concepción de educación y de extensión, también se puso en evidencia la problemática que surge del recambio constante de los voluntarios y colaboradores. Cada principio de año se propone un lugar, una dinámica, un dispositivo del que se desprenden construcción de aprendizajes y saberes; y se abren las convocatorias para integrar los equipos: el recambio nos obliga a empezar de nuevo. Con el tiempo, y luego de problematizar-nos consideramos que esta dinámica podría formar parte de la lógica del espacio. Si bien los voluntarios y colaboradores recambian constantemente así también lo hacen en su rol de estudiantes en la Universidad. Entonces nos preguntamos: ¿podemos considerar esta dinámica como parte de la lógica funcional del espacio? Suponemos que si estamos proponiendo una construcción dialógica debemos pensar: ¿qué se juega en estos vínculos? Vemos que es necesario trabajarlos, darles un marco institucional en este proceso. Desde los aportes teórico-prácticos, el tránsito - tanto de los colaboradores como de los niños y niñas que participan - no resultaría una contradicción sino parte de la dinámica del dispositivo. No obstante, lo que sí consideramos una problemática a trabajar es el abandono de los estudiantes y niños y niñas participantes. Si bien en muchas ocasiones el abandono puede estar causado por circunstancias situacionales o personales, lo cual podría considerarse como funcional al espacio, lo problemático sería lo que Dávila de León (2008) llama "abandono disfuncional", que consiste en las razones controlables que podrían depender del tipo de gestión que deriva en desmotivaciones o insatisfacciones con el funcionamiento del dispositivo o con la lógica de la organización. Es por esta razón que en 2016 implementamos una serie de encuentros al inicio y en el transcurso del año, donde se plantean las inquietudes, expectativas y se pone de manifiesto los modos de construcción y participación del espacio.

Otro hecho a destacar en este último año fue la incorporación de la cátedra de "Prácticas No Formales" de la carrera Licenciatura en Ciencias de la Educación, con el propósito de acercarnos a la curricularización de la extensión. Uno de los principales aportes de estas prácticas fue el acompañamiento de las estudiantes en la planificación mensual de actividades. Este trabajo conjunto conllevó a replantearnos el nombre del dispositivo, que dejó de llamarse "Taller de apoyo escolar" para denominarse "Espacio de aprendizajes y apoyo escolar". Este cambio refleja mejor el quehacer en el espacio, donde ya no solo "hacemos la tarea" sino que se intenta brindar un lugar mucho más amplio en el que se considera al aprendizaje más allá del aula.

El cambio de nombre representa lo que al interior del dispositivo se intenta remarcar, a saber, que no es un maestro particular ni un lugar de trabajo sobre las tareas escolares únicamente; se busca reconstruir dentro del taller y en todo su desarrollo lo que en un principio funcionaba como momento aparte. Ese espacio comodín que fuera pensado dentro del lugar pero desprendido, ahora pasa a ser la lógica de funcionamiento que delimita y articula todo el tiempo dentro del espacio. Es la estrategia lúdico-pedagógica desde la que se abordan todas las actividades y que propone a la actividad creativa como modo de expresión y apropiación para el desarrollo intersubjetivo. 
Siguiendo a Alicia Fernández, consideramos a la modalidad de aprendizaje como una manera personal que tiene cada uno de acercarse y relacionarse con el conocimiento y conformar su saber. Esta modalidad se construye desde el nacimiento y a través de ella nos enfrentamos con la angustia del conocer-desconocer.

"Aprender es apropiarse, apropiación que se da a partir de una elaboración objetivante y subjetivante. La elaboración objetivante permite apropiarse del objeto seriado y clasificándolo, es decir, por ejemplo, reconocer una silla poniéndola en la clase de "silla", es decir, tratando de tomar lo que la iguala a todas las sillas del mundo. En cambio, la elaboración subjetivante tratará de reconocer, de apropiarse de esa silla a partir de aquella única e intransmisible experiencia que haya tenido el sujeto con las sillas." (Fernández, 2010:153)

El aprender es una producción deseante y cognoscente a través de la cual nos vamos construyendo como sujetos. No se circunscribe a lo consciente, ni a lo intelectual, ni a lo escolar. Si bien es cierto que en el proceso de enseñanza-aprendizaje existe transmisión de contenidos (información) para que el aprendiente pueda construir conocimientos a partir de las informaciones necesita significarlas, en un trabajo de implicación desde su saber.

Por eso cuando recordamos a nuestros maestros de la infancia, no lo hacemos por los contenidos enseñados, sino que guardamos inscripto dentro nuestro la modalidad propia de aprendizaje, el posicionamiento aprendiente que esos docentes nos posibilitaron. Es así que entendemos al proceso de enseñanza y aprendizaje como un espacio de identificación, de construcción de procesos identificatorios.

En este sentido, Fernández sostiene que

"enseñar y aprender no son dos procesos complementarios como los que se establecen entre un educador y un educado. Entre el enseñar y el aprender se abre un campo de sorpresa, de lo nuevo, un campo de diferencias" (155).

Si concebimos a la educación como una práctica social, podremos comprender mejor la importancia de la educación no formal. La educación no puede circunscribirse a un sólo "objeto institución" (Fernández, 1994) como lo es la escuela, ya que lo disciplinar, la rigidez horaria, el cumplimiento de ritos y hábitos que ella impone, corre a veces al sujeto aprehendiente del lugar de participante activo en el proceso de enseñanza y aprendizaje.

En el estudio de la educación formal y no formal se requiere, fundamentalmente, una síntesis ya que ambas no solo establecen relaciones de continuidad sino además de complementariedad. Colocar a la educación formal y a la de carácter no formal en posiciones de rivalidad, y de antagonismo acorta las dimensiones que una sociedad tiene para transmitir su legado cultural. La educación no formal puede adoptar funciones de suplencia de la escuela, pero ello no descalifica a ésta última en su función socializante e integradora.

Es entonces que la educación, desde el punto de vista de sus efectos, es un proceso holístico y sinérgico. Un proceso cuya resultante no es la simple acumulación o suma de las experiencias que vive el sujeto, sino la combinación de cómo tales experiencias se influyen mutuamente; interdependencia que se expresa sincrónica y diacrónicamente en los entornos educativos en los que se participa.

A la educación no formal se la ubica en un ámbito espacial que puede o no ser determinado y durante mucho tiempo se ha caído en el error de afirmar que únicamente es la escuela la que educa. Ahora bien, partimos del concepto de que todo patrimonio cultural debe y puede ser apropiado: tal afirmación nos reposiciona frente a la educación no formal. Es por ello que la proclamamos como uno de los tantos espacios destinados a transmitir las dimensiones fundamentales de la cultura: la transmisión del patrimonio cultural dentro de la sociedad es responsabilidad de toda institución educativa ya sea formal o no formal.

Como resume Paulo Freire (1997) en la contratapa de su libro Política y educación, la educación "es un proceso de conocimiento, formación política, manifestación ética... es una práctica indispensable y específica de los seres humanos en la historia como movimiento y como lucha".

Pensamos que la educación no formal puede significar la construcción de escenarios diversos que impliquen la integración y aceptación de las diferencias, contribuyendo a esta propuesta. La actual complejidad de las instituciones que formaron parte del proyecto moderno (familia, Estado, escuela) ha empujado a la sociedad toda a generar nuevos ámbitos para la transmisión de los bienes culturales. Por tal motivo es que hay que considerar también a la educación no formal como espacio destinado a transmitir cultura, a educar. Por eso seguimos la concepción de educación freiriana entendiéndola como un "acto pedagógico y político" (Freire, 1970) ya que refiere a que toda educación, formal o no formal, tiene en el fondo una opinión política, y orientaciones ideológicas que organizan las prácticas. A su vez, Freire sostiene que en todo acto educativo se necesita de un educador que sea revolucionario, que debe ir más allá de la educación tradicional, de la "educación bancaria" como él la llama. En función de la práctica de cada educador realiza los siguientes aportes:

"Su acción, al identificarse, desde luego, con la de los educandos, debe orientarse en el sentido de la liberación de ambos. En el sentido del pensamiento auténtico y no en el de la donación, el de la entrega de conocimientos. Su acción debe estar emparentada con una profunda creencia en los hombres. Creencia en su poder creador". (1970:83) 


\section{Planteos teóricos y desafíos actuales}

Estamos convencidos de que los espacios no formales deben tener presencia tanto de los vecinos como de la sociedad en su conjunto, para que la Universidad y los espacios de formación universitaria dejen de ser claustros cerrados en sí mismos y así poder integrar la práctica de extensión con las de docencia e investigación. Para romper con el academicismo desvinculado, descontextualizado de la realidad social, la Universidad y los estudiantes deben ser partícipes en los cambios que la comunidad procura y demanda, siendo parte. Imaginamos una Universidad atenta a las acciones sociales que logre anticiparse a las necesidades de la comunidad, para que las demandas específicas puedan ser canalizadas por parte de un actor social. Es decir, una Universidad comprometida con el medio y el contexto, implicando y transmitiendo el compromiso social de docentes, estudiantes, y todo actor universitario, para con la comunidad que la sostiene y de la que es parte.

Este propósito no es tarea fácil. Nos encontramos con que las convocatorias no tienen la llegada o la repercusión esperada, a pesar de tener miles de estudiantes; que los docentes no están habituados a pensar en prácticas comunitarias como un terreno posible de formación y trabajo, acostumbrados a reproducir el modelo áulico como único lugar habilitado a la práctica docente; o que los equipos institucionales tienen modos de ser y de hacer instalados y herméticos, o por el contrario cambiante y con resoluciones apresuradas dependientes de disponibilidades y recursos. Las críticas en cualquier caso son producto del choque con la realidad y más que desanimarnos nos desafían a pensar y articular más profundamente, teniendo sumo cuidado en el sentido de la práctica extensionista que queremos sostener y promover desde la gestión.

Actualmente estamos en un proceso de curricularización de la práctica extensionista, en el que se propone visualizarla y valorizarla descartando el presupuesto de que los conocimientos producidos en la academia deben ser transferidos a la sociedad. El fin de legitimar el trabajo en terreno, los conocimientos y valores de aquellos ciudadanos no universitarios, reconociendo los saberes que se traen y los que se producen fuera de las aulas y de los edificios de la Universidad.

Retomando el actual planteo que la Universidad se está haciendo a sí misma y poniendo de relieve que la extensión universitaria va tomando cada vez más una perspectiva integradora es que intentamos guiar las diversas prácticas, en cualquiera de los espacios de participación, bajo la concepción de "integralidad". Es a través de ella que se modifica el acto educativo, porque lo cotidiano y lo concreto se da con estudiantes en terreno y con actores sociales reales; y de esta manera se establece la posibilidad de que exista un diálogo abierto en el acto educativo, más allá del aula, donde los contenidos no son previamente pautados sino que los establece el trabajo concreto que se hace en el territorio. Este modelo, tal como lo plantean Tommasino y Rodríguez (2011) en el Cuaderno de Extensión 1. Integralidad: tensiones y perspectivas, implica una enseñanza activa unida a una práctica en situaciones reales que aumenta la posibilidad de apropiarse del acto educativo. No estamos negando que en el modelo áulico se pueden construir procesos activos, pero estamos seguros de que el trabajo en terreno y la extensión en esta dimensión aportan un plus; y la relación dialógica con los sujetos de la comunidad es ese plus. A este respecto, y en coincidencia con dichos autores, sostenemos que:

"De este modo el acto educativo integral, sostenido en la complementariedad de los actores involucrados, provoca que el lugar de liderazgo no esté siempre depositado en un solo sujeto, no esté solidificado en una persona o figura en particular, que en general es el docente. Por el contrario, se produce una especie de rotación habilitada por la situación concreta de trabajo en el medio". (Tommasino y Rodríguez, 2011:31)

Aquí podemos destacar que los modos de intervención que nos proponemos abordar en cualquiera de los espacios, territorios e instituciones, tienen como eje posibilitar la socialización, habilitando a la construcción histórica de cada sujeto, a la problematización de aquello que perturba, a la reconstrucción de capacidades, significaciones y expectativas, dando lugar a la construcción de lazos sociales. De este modo apostamos a que la práctica extensionista no sea una intervención asistencial ni hegemónica, de aquellas que obturan al sujeto: deben ser intervenciones construidas en conjunto. Muchas veces nos encontramos ante intervenciones realizadas en espacios comunitarios en donde las definiciones sobre las problemáticas que los afectan son realizadas por equipo que las planifica. Por el contrario, consideramos que en la práctica comunitaria las problemáticas deben construirse colectivamente, en donde las personas sean consideradas sujetos activos y no como objetos de observación.

Ante lo anteriormente mencionado y considerando que el contexto actual presenta grandes retos a las universidades públicas como al tipo de conocimiento que en ellas se produce, es que en nuestras prácticas no podemos dejar de preguntarnos: ¿qué tipo de extensión universitaria sostenemos? Las respuestas posibles nos permiten replantear el accionar del estudiantado, de los equipos docentes, investigadores y extensionistas.

Suponemos que nos acercamos a las concepciones a las que hace referencia Boaventura de Sousa Santos cuando renueva la idea de extensión y educación. Esta perspectiva nos marca que la universidad debe participar de manera activa en la construcción 


\section{G6}

Los dispositivos, estrategias y modalidades

de acción necesariamente debemos pensarlos

desde la interdisciplina y la integralidad,

ya que de por sí las problemáticas no son

disciplinadas

de la cohesión social, en la profundización de la democracia, en la lucha por la inclusión social, el cuidado del medio ambiente y en la defensa de la diversidad cultural. Las prácticas extensionistas pueden estar destinadas al trabajo con grupos sociales populares y sus organizaciones, movimientos sociales, comunidades locales o regionales, gobiernos locales; lo importante es que la Universidad pueda estar atenta a problemas reales y actuales de interés social. Al creciente proceso de transnacionalización de la educación superior que trae consigo el proyecto de transformar la universidad en un centro de investigación-acción al servicio del capitalismo global, se le pone como alternativa lo que de Boaventura de Sousa Santos define como la ecología de saberes que consiste en

"la promoción de diálogos entre el saber científico y humanístico que la universidad produce y los saberes legos, populares, tradicionales, urbanos, campesinos, provincianos, de culturas no occidentales que circulan en la sociedad". (2007:67)

En otras palabras, se revaloriza el saber puesto en acción, a partir de una nueva metodología que privilegia la interactividad sobre la unilateralidad y propone un intercambio de conocimientos. Esta alternativa propone un diálogo de los diferentes saberes que, a la hora de pensar en acciones e intervenciones, que deberá apostar por una forma de construir conocimientos que garantice el mayor nivel de participación a los grupos sociales involucrados. Este abordaje permite convertir a la Universidad en un espacio público de "interconocimiento" donde los ciudadanos y los grupos sociales pueden intervenir sin la posición exclusiva de aprendices.

"De este modo la «ecología de saberes» se aleja de una visión idealizada del saber popular o del saber científico, tendiendo a que el diálogo y preferencia de cada uno de ellos esté mediatizada por la acción concreta, por la resolución de problemáticas en conjunto con los actores sociales".

(Tommasino y Rodríguez, 2011:37)
Esta es la forma en que nosotros como SEU y Universidad pretendemos concebir al estudiante y a su formación, es la forma en que pensamos al sujeto, porque este no es, no actúa y no aprende de manera fragmentaria, sino que, por el contrario, lo hace de manera integral. Consideramos a la educación integral como propuesta en la que se piensa a la integralidad del otro y con otros, y desde la que tenemos que diseñar los dispositivos y las estrategias. Es así que todas las actividades y trabajos que desarrollamos desde Área de Programas Comunitarios y la Secretaría tienen como eje el concepto de integralidad, teniendo en cuenta que no es una solución en sí misma, sino que es la consigna que nos convoca. Los dispositivos, estrategias y modalidades de acción necesariamente debemos pensarlos desde la interdisciplina y la integralidad, ya que de por sí las problemáticas no son disciplinadas. Tomando el concepto de intervención de Carballeda, quien la considera como "una forma de acercarse al territorio a través de diferentes dispositivos, instrumentos y modalidades de acción" (2008:17). Nos proponemos concebir a las intervenciones como acciones que tienen una intención, que deben ser planificadas, no disciplinadas, con referencia a un sentido que habilite una lectura de lo que acontece. Acciones que nos permitan posicionarnos frente y junto a otros. Debemos preguntarnos, cuestionarnos durante los procesos de planificación de las intervenciones, y las preguntas deben reformularse cada vez, ante y con cada población y ante cada dinámica o modalidad de intervención, lo cual nos posibilitará argumentar nuestras prácticas y darles un sentido que habilite a la inscripción social, cultural y a la producción subjetiva.

Las modalidades, formas e instrumentos pueden variar mucho. Destacamos que las demandas de la comunidad son un aporte indispensable al momento de pensar en las intervenciones. Cuando las necesidades de la comunidad nos demandan, nos convocan a pensar en dispositivos, debemos estar atentos y propiciar la participación activa para no obstruir. Los vínculos y las relaciones 
que se generan en y con la comunidad permiten reconocer colectivamente problemas y necesidades; identificar posibles soluciones y apostar a la autonomía y el protagonismo de los sujetos participantes. La problematización de la demanda supone, a la vez, un análisis crítico de las situaciones que habilitan procesos interactivos y evitan caer en lo normativizante. El gran desafío es integrar, tanto a la Universidad en sí misma, es decir al interior de esta, así como a la Universidad con el afuera, con la sociedad. Frente a este desafío lo que se propone es una apertura hacia las poblaciones más relegadas, donde los espacios de formación se definen en el lugar en el que se implementa una propuesta de aprendizaje, con lo cual se vuelve a abrir el campo de acción y práctica más allá de lo espacios tradicionales asociados al aula. La formación desde la integralidad es el puntapié para el cambio hacia y desde el interior de las universidades. Esta perspectiva tiene que atravesar las formas de articular las funciones y las acciones de todas las instancias, pensando en que desde los dispositivos existentes se pueden crear otras formas de relacionarse con los conocimientos a partir de otras maneras de participar.

\section{A modo de conclusión...}

A partir de todo este recorrido, concluimos que hemos construido un nuevo posicionamiento teórico referido a las concepciones críticas de las prácticas extensionistas que tienen como eje principal la educación transformadora. Es decir, concebimos a la extensión como un proceso crítico, dialógico y político en que la idea es trascender la formación meramente técnica de la universidad y así lograr procesos de formación integral; esto implica sostener que la educación pública genere universitarios comprometidos con los procesos de transformación social, contribuyendo a la organización y la autonomía de los sectores populares vulnerados.
Estos planteos se complementan con la concepción de educación que se encuentra en la contratapa del libro de Paulo Freire Política y Educación (1997), donde él sostiene que esta "es un proceso de conocimiento, formación política, manifestación ética... es una práctica indispensable y específica de los seres humanos en la historia como movimiento y como lucha".

En este sentido, consideramos que el Espacio de aprendizajes y apoyo escolar en la Biblioteca Popular Pocho Lepratti es un espacio de educación no formal. La actual complejidad de las instituciones que formaron parte del proyecto moderno (familia, Estado, escuela, universidad) ha empujado a generar nuevos ámbitos para la transmisión de los bienes culturales. Por tal motivo es que hay que considerar también a la educación no formal como espacio destinado a transmitir cultura, a educar.

Es así que pensamos que la educación no formal puede significar la construcción de escenarios diversos que impliquen la integración y aceptación de las diferencias, contribuyendo a esta propuesta. Es posible establecer así relaciones de continuidad y de complementariedad junto a la educación formal.

Este posicionamiento debe verse reflejado en las prácticas que sostenemos al interior del Espacio de aprendizajes y apoyo escolar; aquí se intenta remarcar que no queremos reproducir las prácticas formales desarrolladas en la escuela, es decir que no es solo un lugar de trabajo sobre las tareas escolares; sino que se busca reconstruir el espacio comodín, pero ya no pensado como momento aparte al final de cada día como en un principio, sino como parte del desarrollo del taller. Ahora pasa a tener una lógica de funcionamiento que delimita y se articula todo el tiempo dentro del espacio, como parte de la estrategia lúdico-pedagógica y abordando todas las actividades con esa perspectiva que propone la actividad creadora como modo de expresión y apropiación para el desarrollo intersubjetivo. Concordamos con Winnicott cuando plantea al "juego como elaboración imaginativa en torno 
de las funciones corporales, a la relación con los objetos y con la angustia" (2015:80) ya que a través del juego como actividad creadora, el niño se ocupa de la realidad exterior y "puede expresar, mediante materiales externos, las relaciones y angustias internas" (80). Por esto y porque, como sostiene Fernández (2010), el proceso de enseñanza y aprendizaje es un espacio de identificación, de construcción de procesos identificatorios; es que el juego es la principal herramienta y bisagra para la construcción de saberes y aprendizajes significativos.

Podemos concluir entonces, que dentro del espacio se intenta generar un diálogo de saberes, un intercambio y construcción de conocimientos tendiendo a la resolución de problemáticas en conjunto entre todos los actores sociales, los niños y niñas, la organización social, los estudiantes y colaboradores del espacio. De esta manera podemos vincular al espacio con el concepto de "ecología de saberes" propuesto por De Sousa Santos, ya que en este intercambio de saberes puede aterrizar en el aula de la escuela y de la universidad, en el patio, en la vereda, en el barrio, en el territorio.

Todo lo propuesto es parte de un camino del quehacer extensionista en el que seguimos intentando que las prácticas territoriales estén valorizadas curricularmente, dado que consideramos que la excelencia académica no es pertinente sin compromiso social. Por esta razón, en el recorrido de este trabajo damos cuenta de las complejidades de este quehacer en el que aún faltan cátedras que propongan y articulen sus contenidos en contexto. Es decir que este camino no está cerrado sino abierto a la construcción de diversos espacios de prácticas integrales que transversalicen la extensión, la docencia, y la investigación como ejes o pautas históricas de la Reforma Universitaria.

\section{Referencias bibliográficas}

Arocena, R.; Tommasino, H.; Rodríguez, N.; Sutz, J.; Álvarez Pedrosian, E. y Romano, E. (2011). Integralidad: tensiones y perspectivas. Cuadernos de Extensión № 1. Montevideo: Comisión Sectorial de extensión y actividades en el Medio-CSEAM. Carballeda, A. (2004). Capítulo 1. En La intervención en lo social. Buenos Aires: Paidós.

(2008). Capítulo 4. En Los cuerpos fragmentados. Buenos Aires: Paidós. Dávila de León, M. C. (2008). Abandono del voluntariado. Tasas de abandono y causas más frecuentes. Comunicación e ciudadanía. Madrid. Recuperado de: http://eprints.ucm.es/40587/

De Sousa Santos, B. (2007). La Universidad en el Siglo XXI. Bolivia: Plural Editores. Duschatzky, S. (2003). Identidades juveniles, escuela y expulsión social. Buenos Aires: FLACSO.

Fernández, A. (2010). La Inteligencia atrapada. Abordaje psicopedagógico clínico del niño y su familia. Buenos Aires: Nueva Visión.

Fernández, L. (1994). Instituciones educativas. Dinámicas institucionales en situaciones críticas. Buenos Aires: Paidós.

Freire, P. (1970). Pedagogía del oprimido. Uruguay: Tierra Nueva.

- (1987). La Educación para la práctica de la libertad. México: Siglo XXI Editores.

(1997). Política y educación. Brasil: Siglo XXI Editores.

Koldorf, A.; Castro, R. y Tessore, V. (2008). El barrio como espacio de interacción social. IX Congreso Argentino de Antropología Social. Facultad de Humanidades y Ciencias Sociales-Universidad Nacional de Misiones, Posadas. Recuperado de: http://www.aacademica.org/000-080/110

Tommasino, H. y Cano A. (2016). Modelos de extensión universitaria en las universidades latinoamericanas en el siglo XXI: tendencias y controversias. Universidades, 67. México: UDUAL. Winnicott, D. (2015). Notas sobre juego. Manuscrito sin fecha en Exploraciones psicoanalíticas I. Biblioteca fundamental de las ciencias de la psicología. Posfreudianos. Buenos Aires: Paidós. 